\title{
Relação entre comportamento moral dos pais e dos filhos adolescentes ${ }^{1}$
}

\author{
Relation between parents' and \\ adolescents' moral behavior
}

\author{
Laísa Weber PRUST ${ }^{2}$ \\ Paula Inez Cunha GOMIDE ${ }^{3}$
}

\begin{abstract}
Resumo
A moralidade foi abordada neste estudo como uma das variáveis correlacionadas ao aparecimento de comportamentos anti-sociais. A literatura revisada indicou a presença de culpa, vergonha, empatia, honestidade, justiça e generosidade, opiniões positivas sobre o trabalho e ausência de práticas anti-sociais como determinantes do comportamento moral. O objetivo deste estudo foi avaliar se havia diferença entre escores de comportamento moral de famílias de risco e de não risco. O Questionário de Comportamento Moral foi respondido por dois grupos, um de risco e outro de não risco. Cada grupo foi formado por 30 adolescentes e seus pais; os dados propiciaram o cálculo do Índice de Comportamento Moral. O teste não-paramétrico de Maan-Whitney mostrou que as médias dos escores do Índice de Comportamento Moral para famílias de não risco e de risco são estatisticamente diferentes, e o teste de correlação de Spearman verificou que os escores dos adolescentes no Índice de Comportamento Moral correlacionam-se positivamente com os escores dos pais e mães.
\end{abstract}

Uniterms: desenvolvimento moral; práticas educativas parentais; comportamento moral.

\begin{abstract}
Morality, among the variables related to the development of antisocial behavior, was particularly approached in this study. The reviewed literature demonstrates that the presence of guilt, shame, empathy, honesty, generosity and positive opinions about working and absence of antisocial behavior determine moral behavior. This paper evaluated the moral behavior levels difference in two groups: Risk and Non-Risk of families. The Moral Behavior Questionnaire, containing the above-mentioned variables, was drawn up to to assess this relation. This questionnaire was the base for the Moral Behavior Level calculation. Two distinct groups have filled it out (Risk Group and Non-Risk Group). Each group was made up by 30 adolescents and their parents. The non-parametric test of Maan-Whitney showed that the Moral Behavior Level meaning for both groups were different. The correlation analysis of Spearman showed that there was positive correlation between the adolescents' and their parents'Moral Behavior Levels.
\end{abstract}

Uniterms: moral development; parental practices; antisocial behavior.

$\operatorname{cove}$

1 Artigo elaborado a partir da dissertação de L.W. PRUST, intitulada "Efeito do comportamento moral dos pais sobre o comportamento moral dos filhos adolescentes". Universidade Federal do Paraná, 2004.

2 Universidade Federal do Paraná, Programa de Pós-Graduação em Psicologia da Infância e da Adolescência, Departamento de Psicologia, Setor de Ciências Humanas, Letras e Artes. Curitiba, PR, Brasil.

3 Faculdade Evangélica do Paraná, Curso de Psicologia. Rua Padre Anchieta, 2770, 80730-000, Curitiba, PR, Brasil. Correspondência para/Correspondence to: P.I.C. GOMIDE. E-mail: <pgomide@onda.com.br>. 
A freqüência com que são noticiados os envolvimentos de adolescentes em atos anti-sociais justifica a relevância de pesquisas que visam ao conhecimento mais profundo dos fatores que influenciam adolescentes a comportarem-se de maneira inadequada. Esses estudos poderão ser úteis para o desenvolvimento de programas sociais com fins profiláticos. Uma das maneiras de contribuir para isso é examinar as relações entre os estilos parentais e as variáveis envolvidas na pró-sociabilidade.

Este estudo abordou particularmente a moralidade e sua relação com as práticas parentais. Existe correlação entre estilos parentais e o comportamento moral pró-social da criança e do adolescente? Respostas apropriadas para essa questão poderão ajudar os pais a educarem seus filhos de modo a desenvolver mais eficazmente comportamentos morais em sua prole.

A literatura tem apontado o sentimento de culpa, vergonha e empatia, ações honestas, justas e generosas, ausência de práticas anti-sociais, de uso de álcool ou drogas e crenças positivas sobre o trabalho como importantes determinantes do comportamento moral (Araújo,1999; Bandura, 1999; Comte-Sponville, 2000; Davis, 1983; Gewirtz \& Peláez-Nogueras,1991; Gomide 2003, 2004, 2006; Hoffman, 1975, 1979; Hoffman \& Saltzstein, 1967; Loos, Ferreira \& Vasconcelos, 1999; Schlinger, 1995; Smith, Webster, Parrott \& Eyre, 2002; Weber, 2004).

A moralidade é predominantemente inspirada em teorias cognitivistas universalistas como a de Piaget (1932/1977), que considera os atos pró-sociais resultantes do desenvolvimento maturacional do indivíduo, além de outros aspectos, como as interações sociais com menor ênfase nos eventos ambientais antecedentes ou conseqüentes do comportamento. De forma diversa, a perspectiva comportamental (Gewirtz\& PeláezNogueras, 1991; Schlinger, 1995) entende o comportamento moral, assim como qualquer outro comportamento, como produto de uma interação contínua entre o indivíduo e o meio ambiente. A história pessoal faz com que o indivíduo aja de determinada maneira, produzindo uma rede de relações com o ambiente, que conseqüentemente influencia a maneira como responderá ante os diversos estímulos. Assim, a relação entre homem e mundo pressupõe uma transformação recíproca, a qual garante que cada pessoa seja única na 54 sua maneira de agir (Viega \& Vanderberghe, 2001).
A teoria comportamental apresenta duas vertentes de estudo sobre moralidade predominantemente relevantes: a Teoria da Aprendizagem Social (Bandura, 1969/1979, 1999) e a Análise do Comportamento (Skinner, 1974/1982, 1953/1989). Bandura (1969/1979) considera impossível explicar a aquisição de novos comportamentos morais unicamente a partir do processo de reforçamento e punição ou do reflexo condicionado. Seus trabalhos enfatizavam o papel essencial da aprendizagem para o desenvolvimento de comportamentos pró-sociais, vitais para a vida em sociedade. A imitação (Bandura \& Huston, 1961; Bandura, Ross \& Ross, 1961) esteve entre os temas principais de estudo da teoria da aprendizagem social, a qual adota parâmetros relacionais para analisar a moralidade: fatores pessoais (pensamentos morais e reações afetivas) e fatores ambientais atuam influenciando-se bidirecionalmente.

Skinner (1974/1982) atribui o comportamento moral e ético às contingências ambientais. Para ele, uma pessoa age moralmente e consideramos moral porque ela vive num tipo particular de ambiente, e não por ser possuidora de virtudes e consciente de seus semelhantes e de seus direitos.

Do ponto de vista skinneriano, ao analisar a moralidade, especial atenção deve ser dispensada ao comportamento regido ou governado por regras. A expressão "regido ou governado por regras" é comumente empregada para se referir a respostas instaladas ou mantidas sob controle de antecedentes verbais, sob a forma de instruções ou descrições de contingências, enquanto a expressão "controlado por contingências" refere-se em especial a respostas instaladas ou mantidas por suas conseqüências diretas (Nico, 2001a). Grande parte dos comportamentos morais é governada por regras, afinal a maioria dos indivíduos não passou por experiências negativas, tais como problemas com a lei, abuso de álcool ou drogas, e, mesmo sem ter cometido uma ação intensamente injusta ou desonesta, pode inferir que sentiria culpa ou vergonha se fosse responsável por ela.

A comunidade verbal fornece muito cedo no processo de socialização da criança uma infinidade de convenções sobre o que é certo e o que é errado. A possibilidade de descrever contingências e de responder sob controle dessas descrições permite que um indivíduo influencie outro a emitir respostas que podem ser reforçadas positiva ou negativamente, antes que as 
conseqüências diretas dessas respostas possam operar, fortalecendo-as. A partir do mesmo processo, pode-se esperar que o indivíduo deixe de emitir respostas indesejadas, mas muito fortes no seu repertório, ou que poderiam causar dano (Amorim \& Andery, 2002).

O autocontrole é outro aspecto relevante em relação à moralidade. Segundo Skinner (1953/1989), autocontrole se caracteriza pela possibilidade de o próprio indivíduo controlar seu comportamento, sendo capaz de fazer algo a respeito das variáveis que o afetam, isto é, de manipular as variáveis das quais o comportamento é função. Para Nico (2001b), o autocontrole será tanto mais necessário quanto maior for o conflito entre as contingências de reforço.

A dinâmica do grupo familiar é muito poderosa no processo de desenvolvimento da criança, pois é em casa que adquirirá quase todos os repertórios comportamentais básicos. Pais sensíveis, responsivos e pró-sociais exercem forte influência no desenvolvimento do senso de cooperação e reciprocidade das crianças despertando expectativas positivas sobre a disposição dos outros para dar e receber ajuda (Koller \& Bernardes, 1997). Pode-se afirmar que os pais ou cuidadores representam a primeira e maior fonte de reforçamento e modelo para os filhos.

O comportamento moral como prática educativa é entendido por Gomide $(2001,2004)$ como o processo de modelagem de papéis sociais no que se relaciona principalmente à transmissão de normas e valores mediante modelo fornecido pelos pais. Ao interagirem com seus filhos de maneira afetuosa e empática, ao explicitarem sua opinião, aprovação ou desaprovação das situações vividas por eles próprios, pelos filhos ou por outras pessoas, ao responsabilizarem-se e repararem um dano causado a alguém, os pais estarão oferecendo modelos de valores esperados no ambiente familiar e que serão generalizados em outras circunstâncias.

Estudos empíricos têm corroborado a relação entre os estilos parentais e o comportamento moral. Diferenças entre a qualidade da relação afetuosa entre pais e as práticas parentais disciplinares têm sido associadas com a sofisticação de raciocínio moral de crianças (Dunn, Brown \& Maguire, 1995). Pesquisadores encontraram correlação positiva entre comportamento pró-social de crianças e práticas maternas de educação voltadas para o desenvolvimento da empatia (Eisenberg et al., 1993). Outros estudos apontam menor vulnerabilidade ao uso de drogas em adolescentes apegados à figura paterna, lar cujo ambiente é saudável, aceitação de crenças sobre o bom comportamento e desaprovação paterna específica a certos comportamentos (Nurco \& Lerner, 1996). Patterson, Reid e Dishion (1992) apontam o uso apropriado de reforçamento positivo, a habilidade para resolver problemas de grupo, a supervisão e a monitoria dos pais como importantes fatores relacionados à pró-sociabilidade.

Por meio de estudo empírico, Wright e Cullen (2001) confirmaram os resultados de pesquisas anteriores que indicavam que o controle desempenha papel central para a etiologia de comportamentos anti-sociais. Práticas parentais como supervisão, estabelecimentos de limites, posicionamentos claros em relação a regras e relacionamento estreito entre os membros da família podem reduzir a possibilidade de engajamento em comportamentos de risco. Embora o controle seja uma importante variável a ser considerada diante de comportamentos anti-sociais, os autores ressaltam também a necessidade de incluir o suporte ou apoio nos estudos sobre o tema. Segundo eles, embora sejam variáveis distintas, suporte e controle estão relacionados e seriam algumas das características de pais cujas práticas inibem o desenvolvimento de comportamentos anti-sociais.

Comportamento anti-social é definido como todo aquele que causa prejuízo a si ou ao outro, incluindo aí a mentira, a piromania, a pixação de patrimônio, a evasão escolar, o uso de drogas, o comportamento agressivo, além dos delitos leves e graves (Gomide, 2003). Já comportamento pro-sócial é aquele que desenvolve as virtudes, como, por exemplo, o comportamento altruísta, como a justiça, a solidariedade, a generosidade, a empatia, a gratidão, etc. (La Taille, 2000). Dessa forma, as famílias de risco são aquelas que têm índices altos de comportamentos anti-sociais e, conseqüentemente, baixos de comportamentos pró-sociais (Gomide, 2003).

O uso de álcool pelos pais e sua relação com o desenvolvimento de comportamento anti-social nos filhos foi estudado por Eiden e Leonard (2000), que verificaram que o alcoolismo, acrescido de características associadas com comportamento anti-social e depressão por parte dos pais, tem papel significativo na predição de possíveis privações afetivas relacionadas ao apego em crianças pequenas, que podem afetar o 
desenvolvimento de relações pró-sociais nas crianças. Nurco e Lerner (1996) apontam o modelo positivo do pai e sua presença na família como inibidores do uso de drogas e álcool em adolescentes.

O trabalho - outra variável relevante - parece ter relação com as práticas educativas utilizadas pelos pais. Uma pesquisa de Kerka (2000) indicou haver influência dos estilos parentais na escolha profissional dos filhos. Filhos de pais autoritários tendem a obter sucesso nos estudos, porém podem escolher a profissão em função do desejo dos pais. Filhos de pais negligentes tendem a ter dificuldade em discriminar seus próprios interesses e, por isso, ficam indecisos quanto à escolha profissional. Pais responsivos, cujas práticas educativas permitiram aos filhos desenvolver maior autonomia para tomar decisões e transmitem valores sobre o trabalho, habilidades de comunicação e de resolução de problemas, tendem a ser mais bem-sucedidos ao encaminhar os filhos para a busca da realização profissional e da independência financeira.

Tendo em vista que o comportamento moral é uma das variáveis que inibem o desenvolvimento de comportamento anti-social, o objetivo deste estudo foi avaliar se havia diferença entre os escores de comportamento moral de famílias de risco e de não risco. Para essa finalidade foi elaborado um Questionário de Comportamento Moral, que abordou as seguintes variáveis: culpa, vergonha, honestidade, justiça, generosidade, empatia, crenças sobre o trabalho, uso de álcool e freqüência de comportamentos anti-sociais.

\section{Método}

\section{Participantes}

Participaram deste estudo 60 famílias, sendo 30 de risco e 30 de não risco. As famílias foram selecionadas como de risco ou não mediante a aplicação do Inventário de Estilo Parental (IEP) (Gomide, 2006) em adolescentes, de ambos os sexos, da 7a e 8a séries do ensino fundamental de escolas públicas e privadas de Curitiba. O grupo de famílias de não risco foi composto por pais que obtiveram índice de estilo parental igual ou maior a +7 para ambos os membros do casal e o grupo de risco foi formado por pais com índice de estilo parental igual ou menor que -3 , para pelo menos um dos membros do casal. À amostra de risco foi incluída a participação de três adolescentes autores de atos infracionais que cumpriam medidas socioeducativas na Vara de Infância e Juventude da Comarca de Curitiba e seus pais.

Os dados das 60 famílias foram coletados em suas residências, tendo sido utilizados dois instrumentos para coleta de dados:

1) Inventário de Estilo Parental (Gomide, 2006), utilizado para selecionar a amostra, é um instrumento que consta de 42 questões que abordam duas práticas educativas positivas e cinco negativas. O índice de estilo parental é calculado somando-se os pontos obtidos nas questões referentes às práticas positivas (monitoria positiva e comportamento moral) que são subtraídos do somatório dos pontos das práticas negativas (punição inconsistente, negligência, disciplina relaxada, monitoria negativa e abuso físico). O índice quando negativo informa a prevalência de práticas educativas negativas e, quando positivo, a presença de práticas positivas no processo educacional. Os adolescentes responderam a dois inventários, um sobre as práticas educativas adotadas pelo pai e outro pela mãe, separadamente.

2) Questionários de Comportamento Moral (elaborados pelos autores deste trabalho), sendo um respondido pelos adolescentes (contendo 27 questões) e outro pelos pais e mães (contendo 22 questões), aplicados individualmente. Cada questão apresentava múltiplas alternativas entre as quais o respondente deveria escolher as que mais se assemelham ao seu comportamento diante da situação proposta. As questões para o adolescente e para os pais eram equivalentes em conteúdo e se relacionavam às seguintes variáveis: honestidade, atos anti-sociais, trabalho, justiça, generosidade, culpa, vergonha, empatia, uso de álcool.

A cada uma das alternativas do questionário foi atribuído um valor não conhecido pelo respondente e que variou de acordo com o grau de adequação da conduta abordada $(+2,+1,0,-2,-1)$ : quanto mais adequada a conduta, maior o valor atribuído e vice-versa. Os valores atribuídos às condutas foram obtidos por meio de julgamento de sete juízes ${ }^{4}$, e possibilitaram o cálculo do índice de comportamento moral (ICM) para 
cada participante, registrado na folha de resposta.

A pontuação de cada questão variava de no mínimo -2 a no máximo +2; portanto se o respondente assinalasse mais de uma alternativa cujos valores somados resultassem maiores que +2 , o valor atribuído à questão continuaria sendo +2 . O mesmo ocorreu com as alternativas com valor negativo. Além disso, se o respondente assinalasse uma alternativa de valor positivo e outra de valor negativo, os valores seriam somados; por exemplo, assinalando uma alternativa de valor +2 e outra de valor -2 , o resultado da questão seria 0 , ou se fosse assinalada uma alternativa +2 e outra de valor -1, o valor atribuído à questão seria +1. Procedendo dessa forma, os valores do ICP variaram de -44 a +44 para os pais e de $-54 \mathrm{a}+54$ para os adolescentes.

\section{Procedimentos}

Os participantes foram selecionados entre os estudantes submetidos ao IEP em pesquisa anterior (Gomide, 2003), e deveriam preencher os critérios estabelecidos na seção "Participantes". A fim de iniciar a coleta de dados, a pesquisadora pediu ao coordenador pedagógico dos colégios onde foi aplicado o IEP permissão para fazer contato com os adolescentes que foram selecionados na amostra, convidando-os a participar da pesquisa. Os adolescentes que aceitaram o convite receberam uma carta endereçada aos pais, tendo sido, posteriormente, agendado um horário para a aplicação do instrumento. Nessa ocasião, os pais e o adolescente foram solicitados a responder ao Questionário de Comportamento Moral, esclarecendo-se que se tratava de uma pesquisa sobre maneiras de educar os filhos. Os pais assinaram o Termo de Consentimento Livre e Esclarecido, que explicava que os dados seriam utilizados somente para pesquisa, resguardado o sigilo apropriado.

\section{Resultados e Discussão}

Foi calculado para cada participante o Índice de Comportamento Moral (ICM) obtido mediante a aplicação do Questionário de Comportamento Moral. Primeiramente, buscou-se verificar se havia correlação entre os ICM dos pais e os ICM dos adolescentes, por meio do teste de correlação de Spearman. Em seguida, por meio do teste de Maan-Whitney, compararam-se os ICM dos membros das famílias de risco com os dos membros das famílias de não risco, a fim de avaliar as possíveis diferenças entre os grupos.

A amostra foi constituída por 30 famílias de não risco e 30 de risco que apresentaram, na média, faixas etárias semelhantes. No grupo de não risco, a idade média das mães foi de 42,3 anos e a dos pais foi de 44,9 anos; a amostra de adolescentes desse grupo foi composta por 18 do sexo masculino e 12 do sexo feminino, com idade média de 13,6. No grupo de risco, a idade média das mães foi de 42,1 e a dos pais de 44,1; a amostra de adolescentes do grupo de risco foi formada por 16 do sexo masculino e 14 do sexo feminino, com idade média de 14,4 anos.

A escolaridade dos participantes da amostra diferiu entre os dois grupos. As mães do grupo de não risco tinham predominantemente nível superior (66,6\%), as demais tinham nível médio (30,0\%), apenas uma (3,0\%) tinha nível fundamental incompleto. Os pais desse grupo apresentaram escolaridade semelhante à das mães, com 66,6\% de nível superior; $26,6 \%$ de nível médio e apenas dois $(6,6 \%)$ com nível fundamental. Entre os adolescentes, 63,3\% possuíam ensino fundamental completo e $36,7 \%$, fundamental incompleto, e apenas 13,3\% deles estavam em defasagem escolar, ou seja, possuíam 15 anos e deveriam estar cursando o ensino médio, mas ainda se encontravam no ensino fundamental.

A escolaridade do grupo de risco mostrou-se rebaixada em relação ao grupo de não risco. Entre as mães, 40,0\% possuíam nível superior; 50,0\% nível médio e 10,0\% nível fundamental. Entre os pais, 36,6\% tinham nível superior; 60,0\% nível médio e apenas 3,3\% nível fundamental incompleto. A maioria, 76,6\% dos adolescentes desse grupo, possuía o ensino fundamental completo e $20,0 \%$ o fundamental incompleto. Porém, 16,6\% tinham escolaridade inferior à esperada para a idade; dois deles, com 16 anos, ainda não haviam ingressado no ensino médio. Os outros três, com 18 anos, ainda não haviam concluído o ensino fundamental. Esses últimos três casos, fortemente discrepantes, se referiam aos adolescentes em conflito com a lei.

O nível de escolaridade rebaixado dos pais do grupo de risco em relação aos de não risco $(66,6 \%$ com nível superior para pai e mãe do grupo de não risco, 
contra 36,6\% e 40,0\% para pai e mãe, respectivamente do grupo de risco) é apontado pela literatura como um fator de risco importante, uma vez que os estudos de Patterson et al. (1992) e de Gallo (2006) apontam para uma correlação positiva entre baixa escolaridade e desenvolvimento de comportamentos anti-sociais.

A fim de estabelecer a existência da correlação entre o ICM dos pais e o ICM de seus filhos adolescentes, aplicou-se o teste de correlação de Spearman com a hipótese nula de que não existia correlação entre os ICM dos membros da família.

Verificou-se que o ICM dos adolescentes correlacionou-se positivamente com os índices dos pais e das mães. A correlação obteve índices mais significativos entre os adolescentes e seus respectivos pais $(r=0,45 ; p<0,001)$ do que entre os adolescentes e suas mães ( $r=0,3 ; p=0,002$ ), porém ambos são significativos estatisticamente, indicando, por um lado, que quando os pais apresentam médias elevadas de comportamento moral, os filhos também as apresentam, e, por outro lado, que quando há baixos ICM entre os pais, o mesmo ocorre com os filhos.

Os estudos de Nurco e Lerner (1996) encontraram que a figura paterna está fortemente relacionada ao desenvolvimento de comportamentos morais na família. Em famílias em que o pai fala o que é certo ou errado e que apresenta bons modelos de comportamentos morais não foram encontrados filhos usuários de drogas. Os dados dos autores não mostraram as mesmas correlações com os comportamentos morais maternos. Poder-se-ia levantar a possibilidade de que o homem em nossa sociedade representa ainda a figura de autoridade dentro da família e que seu comportamento moral exemplar influencia mais o comportamento moral dos filhos do que o da mãe com o mesmo nível de comportamento moral.

O índice de comportamento moral de cada participante foi obtido pelo somatório dos valores atribuídos às respostas dadas ao questionário de comportamento moral que avaliou as seguintes variáveis: honestidade, atos anti-sociais, trabalho, justiça, generosidade, culpa, vergonha, empatia e uso de álcool.

Para avaliar se o índice de comportamento moral dos componentes das famílias de risco era semelhante ao dos componentes das famílias de não risco, escolheu-se o teste não paramétrico de Maan-Whitney, por se tratar de amostra com número de participantes no limite mínimo de análises paramétricas, e por se lidar com categorias comportamentais. Para isso se considerou a hipótese nula de que os resultados do ICM seriam iguais entre os grupos de risco e de não risco, utilizando-se o nível de significância de 5\%.

Os resultados indicaram a rejeição da hipótese nula, ou seja, ao nível de significância de 0,05, o teste não paramétrico de Mann-Whitney mostrou que as médias dos escores do ICM para famílias de não risco e de risco são estatisticamente diferentes, ou seja, na comparação entre os grupos de risco e os de não risco obteve-se para as mães $U=291$ ( $p=0,02$; para os pais $U=216,5(p=0,001)$; para os adolescentes $U=70,5$ $(p<0,001)$ e para a família $U=84(p<0,001)$. Isso significa que as famílias de não risco apresentaram médias de comportamento moral superiores às famílias de risco em todas as categorias (mãe, pai, adolescente e família), com especial diferença entre os adolescentes.

Pode-se observar essas diferenças de forma mais nítida na Figura 1, a qual mostra perfeitamente que a maior diferença encontrada foi entre o ICM dos adolescentes; as diferenças entre os ICM de pais e mães foram de menor amplitude, porém significativas estatisticamente, como já anteriormente descrito.

Segundo a teoria da desejabilidade social, o participante pode responder em desacordo com a realidade, mas de acordo com a resposta que ele acredita que seja desejada pelo investigador (Ellingson, Sackett \& Hough, 1999). Isso pode ter influenciado as respostas

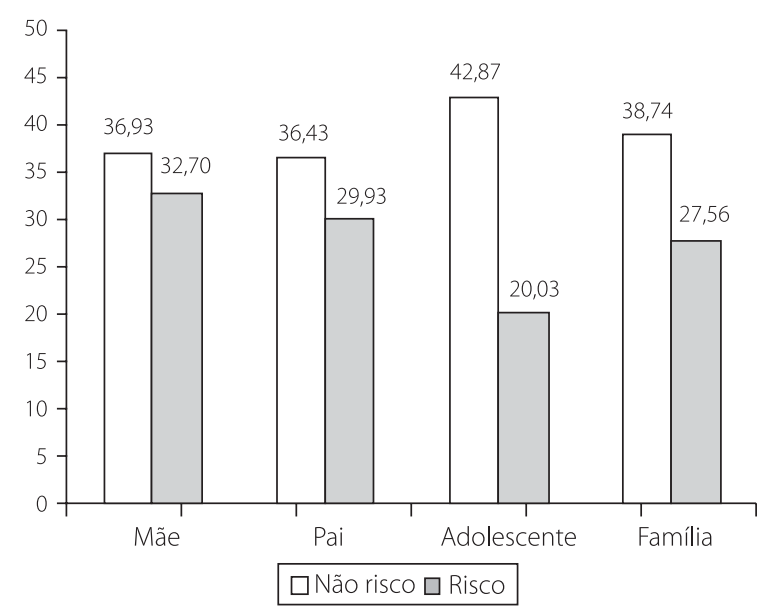

Figura 1. Média dos índices de comportamento moral dos integrantes das famílias estudadas. 
das mães e dos pais da amostra, cujas médias variaram de maneira menos significativa entre os grupos. Entretanto, aparentemente, os adolescentes provavelmente se sentiram mais à vontade para responder ao questionário sem se preocupar com as implicações sociais de suas respostas. Essa é uma hipótese a ser investigada posteriormente.

Pinheiro (2003), avaliando qualitativamente famílias de risco e de não risco, encontrou que pais de risco não tinham argumentos morais e não davam oportunidades aos filhos de experienciarem as virtudes da solidariedade, justiça, empatia; e que pais do grupo de não risco realizavam suas tarefas educativas exercitando a empatia, a autocrítica, a vergonha diante de atos anti-sociais. Tanto os dados de Pinheiro como os desta pesquisa foram obtidos através de entrevistas. Nas duas pesquisas todos os membros das famílias de risco se comportaram com baixos ICM, revelando que sem ensinamentos, modelos fornecidos pelos pais, os adolescentes também não desenvolvem um padrão de comportamento moral adequado. Pinheiro (2003) destaca que alguns pais que tiveram pais com práticas educativas negativas superaram suas dificuldades através de terapias, e afirmaram que procuraram não repetir com os filhos suas experiências. Essa informação é relevante, pois indica a possibilidade de reversão do ciclo da violência remetendo à violência.

\section{Conclusão}

Os estudos da prática educativa denominada comportamento moral estão intrinsecamente ligados ao desenvolvimento dos comportamentos inibitórios dos atos anti-sociais (Carvalho, 2003; Pinheiro, 2003; Weber, 2004). A aprendizagem das grandes virtudes, como salienta Comte-Sponville (2000), é mais bem ensinada através do exemplo do que das palavras.

Este estudo procurou verificar se havia correlação entre o ICM dos pais e de seus filhos e também, ao se comparar dois grupos, um de famílias de risco e outro de famílias de não risco, se havia diferença no ICM. Os dados demonstraram que as médias do ICM dos membros das famílias de não risco foram diferentes estatisticamente e maiores que as dos membros das famílias de risco, ou seja, para as mães (não risco = 36,9; risco $=32,7$ ); para os pais (não risco $=36,4$; risco $=29,97$ ); para os adolescentes (não risco $=42,9$; risco $=20$ ) e, finalmente, utilizando o índice que refletia a média das famílias, para a de não risco $(38,7)$ e de risco $(27,6)$.

O teste não paramétrico de Maan-Whitney mostrou diferenças estatisticamente significativas nos ICM das famílias, como indicado pelas pesquisas de Nurco e Lerner (1996) e de Pinheiro (2003). Um maior nível de comportamento moral entre as famílias de não risco indica que pais que fornecem modelos morais, exercitam a empatia e fazem a reparação do dano diante de atos anti-sociais têm filhos com melhores ICM e que não são usuários de drogas.

O teste de correlação de Spearman permitiu verificar que os escores de ICM dos adolescentes correlacionam-se positivamente com os escores do ICM de pais e mães. Resumidamente, os testes estatísticos de Maan-Whitney e de Spearman apontaram que os membros das famílias agem em relação à moralidade de forma semelhante, ou seja, quando os pais apresentam níveis elevados de comportamento moral, os filhos também os apresentam e vice-versa. Esses dados são bons indicadores de que é preciso que os pais incorporem entre suas tarefas educativas o exercício de atividades que desenvolvam as virtudes, tanto dando modelos apropriados como oportunidades aos filhos para experienciarem situações na quais os valores morais estão presentes.

\section{Referências}

Amorim, C., \& Andery, M. A. (2002). Quando esperar (ou não) pela correspondência entre comportamento e comportamento não-verbal. In H. J. Guilhardi, M. B. B. P. Madi, P. P. Queiroz \& M. C. Scoz (Orgs.), Sobre comportamento e cognição: Vol. 10. Contribuições para a construção da teoria do comportamento (pp.37-48). Santo André: ESEtec.

Araújo, U. F. (1999). Conto de escola: a vergonha como um regulador moral. São Paulo: Moderna.

Bandura, A. (1979). Modificação do comportamento. Rio de Janeiro:Interamericana. (Originalmente publicado em 1969).

Bandura, A. (1999). Moral disengagement in the perpetration of inhumanities. Personality and Social Psychology Review, 3 (3), 193-209.

Bandura, A., \& Huston, A. C. (1961). Identifications as a process of identification learning. Journal of Abnourmal and Social Psychology, 63, 311-318.

Bandura, A., Ross, D., \& Ross, S. A. (1961). Transmission of aggressive through imitation of aggressive models. Journal of Abnourmal and Social Psychology, 63, 575-582. 
Carvalho, M. C. N. (2003). Efeito das práticas educativas parentais sobre o comportamento infrator de adolescentes. dissertação de mestrado não-publicada, Programa de Pós-Graduação em Psicologia da Infância e Adolescência, Universidade Federal do Paraná, Curitiba.

Comte-Sponville, A. (2000). Pequeno tratado das grandes virtudes. São Paulo: Martins Fontes.

Davis, M. H. (1983). Measuring individual differences in empathy: evidence for a multidimensional approach. Journal of Personality and Social Psychology, 44, 113-126.

Dunn, J., Brown, J. R., \& Maguire, M. (1995). The development of children's moral sensibility: individual differences and emotion understanding. Developmental Psychology, $31,649-659$

Eiden, R. D., \& Leonard, K. (2000). Paternal alcoholism, parental psychopatology and aggravation with infants. Journal of Substance Abuse, 11 (1), 17-29.

Eisenberg, N., Fabes, R. A. A., Carlo G., Speer L. A., Switzer G., Karbon, M., \& Troyer, D. (1993). The relations of empathy-related emotions and maternal practices to children's comforting behavior. Journal of Experimental Child Psychology, 55 (2), 131-150.

Ellingson, J. E., Sackett, P. R., \& Hough, L. M. (1999). Social desirability coretions in personality measurement issues of applicant comparison and construct validity. Journal of Applied Psychology, 84, 155-166.

Gewirtz, J., \& Peláez-Nogueras, M. (1991). Proximal mechanisms underlying the aquisition of moral behavior patterns. In W. M. Kurtines \& J. L. Gewirtz (Orgs.), Handbook of moral behavior and development vol 1: theory (pp.153-182). New Jersey: Lawrence Eribaum Associates.

Gallo, A. E. (2006). Adolescentes em conflito com a lei: perfil e intervenção. Tese de doutorado não-publicada, Universidade Federal de São Carlos.

Gomide, P. I. C. (2001). Efeito das práticas educativas no desenvolvimento do comportamento anti-social. In M. L. Marinho \& V. E. Caballo (Orgs.), Psicologia clínica e da Saúde. Londrina: UEL.

Gomide, P. I. C. (2003). Estilos parentais e comportamento anti-social. In A. Del Prette \& Z. A. P. Del Prette (Orgs.), Habilidades sociais, desenvolvimento e aprendizagem: questões conceituais, avaliação e intervenção. Campinas: Alínea.

Gomide, P. I. C. (2004). Pais presentes, pais ausentes: regras e limites. Petrópolis: Vozes.

Gomide, P. I. C. (2006). Inventário de estilos parentais: modelo teórico, manual de aplicação, apuração e interpretação. Petrópolis: Vozes.

Hoffman, M. L. (1975). Moral internalization, parental power, and the nature of parent-child interaction. Developmental Psychology, 11, 228-239.

Hoffman, M. L. (1979). Development of moral thought, feeling and behavior. American Psychlologist, 34, 958-966.

Hoffman, M. L., \& Saltztein, H. D. (1967) Parent discipline and the child's moral development. Journal of Personality and Social Psychology, 5, 45-57.

Kerka, S. (2000). Parenting and career development. ERIC Clearinghouse on Adult, Career, and Vocational
Educational. Washington, DC: Columbus Office of Educational Research and Improvement. (ERIC Digest, no 214).

Koller, S. H., \& Bernardes, N. M. G. (1997). Desenvolvimento moral pró-social: semelhanças e diferenças entre os modelos teóricos de Eisenberg e Kohlberg. Estudos de Psicologia (Natal), 2 (2), 223-262.

La Taille, Y. (2000). Para um estudo psicológico das virtudes morais. Educação e Pesquisa, 26 (2), 109-121.

Loos, H., Ferreira, S. P. A., \& Vasconcelos, F. C. (1999). Julgamento moral: estudo comparativo entre crianças institucionalizadas e crianças de comunidade de baixa renda com relação à emergência do sentimento de culpa. Psicologia: Reflexão e Crítica, 12 (1), 47-70.

Nico, Y. C. (2001a). Regras e insensibilidade: conceitos teóricos, algumas considerações teóricas e empíricas. In R. R. Kerbauy \& R. C. Wielenska (Orgs.), Sobre comportamento e cognição (Vol.4, pp.31-39). Santo André: Arbytes.

Nico, Y. C. (2001b). O que é autocontrole, tomada de decisão e solução de problemas na perspectiva de B. F. Skinner. In H. J. Guilhardi, M. B. B. P. Madi, P. P., Queiroz \& M.C. Scoz (Orgs.), Sobre comportamento e cognição (Vol. 7, pp.62-70). Santo André: ESETec.

Nurco, D., \& Lerner, M. (1996). Vulnerability to narcotic addiction: family structure and functioning. Journal of Drug Issues, 26, 1007-1025.

Patterson, G., Reid, J., \& Dishion, T. (1992). Antisocial Boys. EUA: Castalia Publishing.

Piaget, J. (1977). O julgamento moral da criança. São Paulo: Mestre Jou. (Originalmente publicado em 1932).

Pinheiro, D. N. (2003). Estilo parental: uma análise qualitativa. Dissertação de mestrado não-publicada, Programa de Pós-Graduação em Psicologia da Infância e Adolescência, Universidade Federal do Parana, Curitiba.

Schlinger, Jr. H. D. (1995). A behavior analytic view of child development. New York: Plenum Press.

Skinner, B. F. (1982). Sobre o behaviorismo. São Paulo: Cultrix.

Skinner, B. F. (1989). Ciência e comportamento humano. São Paulo: Martins Fontes. (Originalmente publicado em 1953).

Smith, R. H., Webster, J. M., Perrod, P. W., \& Eyre, H. L. (2002). The role of public exposure in moral and nonmoral shame and guilt. Journal of Personality and Social Psychology, 83 (1), 138-159.

Viega, M., \&Vanderberghe, L. (2001). Behaviorismo: reflexões acerca de sua epistemologia. Revista Brasileira de Terapia Comportamental e Cognitiva, 3, 9-18.

Weber, L. (2004). Efeito do comportamento moral dos pais sobre o comportamento moral dos filhos adolescentes. Dissertação de mestrado não-publicada, Universidade Federal do Paraná, Curitiba.

Wright, P., \& Cullen, F. (2001). Parental efficacy and delinquent behavior: do control and support matter? Criminology, 39 (3), 704-705.

Recebido em: 11/4//2006

Versão final reapresentada em: 13/6/2006

Aprovado em: 13/6/2006 